\title{
Clinical presentation and outcomes of non-typhoidal Salmonella infections in patients with cancer
}

\author{
Nobuyoshi Mori ${ }^{1,3}$, Ariel D. Szvalbb ${ }^{1}$, Javier A. Adachi ${ }^{1}$, Jeffrey J. Tarrand ${ }^{2}$ and Victor E. Mulanovich ${ }^{1 *}$ (i)
}

\begin{abstract}
Background: Non-typhoidal Salmonella (NTS) infection is thought to be more severe in cancer patients, but this has not been studied since the development of new cancer therapies, increasing antibiotic resistance and the introduction of new antibiotics. We sought to describe the demographic characteristics, microbiological findings, clinical manifestations, and outcomes of NTS infections in cancer patients at our institution.

Methods: We reviewed microbiology laboratory records and identified patients who had cancer and from whom NTS organisms were recovered between January 1, 2000 and December 31, 2013, at a comprehensive cancer center in Houston, Texas. Descriptive statistics were used to summarize patient characteristics, clinical presentation and outcomes.
\end{abstract}

Results: We identified 110 isolates from 82 patients with 88 episodes of NTS infection (including five relapses [6\%] in four patients, and two consecutive episodes in one patient). Fifty-five patients (67\%) had hematologic malignancies. Most NTS isolates were susceptible to the commonly prescribed antimicrobials. Sixty-nine percent of patients had sepsis and one-third had severe sepsis or septic shock. Gastroenteritis, bacteremia, or both were present in 69\% of patients, and the rest had focal infection. Mortality at 30 days was low (8\%). Relapses occurred only in patients receiving $\leq 10$ days of antibiotic therapy.

Conclusions: NTS affects predominantly patients with hematologic malignancies, followed by gastrointestinal and genitourinary cancers. Invasive disease, sepsis, and septic shock are common presentations among admitted patients. Antimicrobial prophylaxis may not prevent NTS infection. Thirty-day mortality and attributable mortality rates were low in our series compared to older case series. Early appropriate antibiotic therapy may have had a role in decreasing mortality. Relapses occurred in patients receiving $\leq 10$ days of therapy, suggesting the need for longer duration of antibiotic therapy in cancer patients with uncomplicated NTS infections.

Keywords: Salmonella infections, Foodborne diseases, Febrile neutropenia, Immunocompromised host, Sepsis, Bacteremia

\footnotetext{
*Correspondence: vmulanov@mdanderson.org

${ }^{1}$ Department of Infectious Diseases, Infection Control and Employee

Health, The University of Texas MD Anderson Cancer Center, 1515

Holcombe Boulevard, Unit 1460, Houston, Texas 77030, USA

Full list of author information is available at the end of the article
}

\begin{abstract}
Background
Non-typhoidal Salmonella (NTS) is the leading cause of illness, hospitalizations, and death among foodborne pathogens in the United States [1]. In immunocompetent hosts, NTS infection presents most commonly as diarrheal illness, usually self-limited. Factors that increase the risk for NTS infection include extremes
\end{abstract}


of age, reduced gastric acid secretion due to atrophic gastritis, gastric surgery, or medications (H2 receptor antagonists or proton pump inhibitors), and use of antibiotics in the prior month [2-4]. Immunosuppressed patients are at increased risk of NTS infection, particularly severe disease such as septicemia and disseminated infection [3, 5]. In 1967, Han et al. reported that salmonellosis occurred more frequently in cancer patients than in other patients at his institution [6], and in 1969, Cherubin et al. reported that 41\% Salmonella septicemias in New York City occurred in patients with cancer [7].

Although NTS infection in cancer patients has been described in Southeast Asia [5, 8, 9], the largest case series were published in Europe in 1994 [10] and in the United States in 1971 [11]. Since then, considerable improvements in cancer therapy and supportive care, as well as new antimicrobials and increasing antibiotic resistance, have altered the landscape in which these infections occur. The aim of the present study was to describe the demographic characteristics, prevalence of risk factors previously reported in the literature, microbiological findings, clinical manifestations, and outcomes of NTS infections in patients with cancer in our institution.

\section{Methods}

\section{Hospital setting, bacterial isolates, and data collection}

The study was conducted at a comprehensive cancer center in Houston, Texas, after approval by the Institutional Review Board. Our microbiology laboratory receives and processes all clinical cultures. We therefore reviewed our laboratory records to identify patients from whom NTS organisms were recovered between January 1, 2000 and December 31, 2013. At that time, we had not yet instituted stool testing with a multiplex PCR panel for enteric pathogens. Thus, NTS from these patients was identified from cultures using Vitek-2 or Vitek MS (bioMérieux, Durham, North Carolina). The serogroups of Salmonella isolates were determined by $\mathrm{O}$ antisera (Difco Laboratories, Detroit, Michigan) using the slide agglutination test and confirmed by the Houston Health and Human Services Department.

We retrospectively reviewed the medical records of all patients with cancer who had positive cultures for NTS during the period studied. Data extracted from the medical record included demographic characteristics, cancer diagnosis, other underlying diseases, use of immunosuppressant and chemotherapeutic agents, antimicrobial prophylaxis, clinical manifestations, severity of disease, site of infection, NTS serogroup, antimicrobial susceptibilities, treatment, and outcomes.

\section{Definitions}

Infection with NTS was defined as the presence of clinical manifestations, including systemic and/or localized signs or symptoms, along with recovery of the organism from clinically infected sites such as blood, stool, urine, sputum, or other normally sterile sites. An episode of NTS infection included all positive cultures from any site within a 2-week period. In order to differentiate relapse from persistent infection, re-infection with the same NTS serotype, or asymptomatic carrier state, we defined relapse as a new positive culture(s) with the same NTS serotype occurring $\geq 2$ weeks and $\leq 3$ months from the previous positive cultures in a patient who received therapy with an antibiotic to which the isolate was reported sensitive. Consecutive infections occurred when a patient developed $\geq 2$ episodes of infection with different serotypes of NTS, regardless of the time between them.

Episodes of NTS infection were classified as (1) acute gastroenteritis (GE), if the patient presented with diarrhea (with or without other gastrointestinal symptoms or fever) and had positive stool cultures but no evidence of extra-intestinal organ involvement or positive blood cultures; or (2) invasive disease, comprising three subcategories based on the classification proposed by Ramos et al. [12]: (a) GE with bacteremia, if the patient presented with diarrhea (with or without positive stool cultures) within a month of positive blood cultures but without focal infection; (b) primary bacteremia, when NTS was isolated from blood cultures in a patient without GE or evidence of focal infection; and (c) focal infection, when an organ or system was affected based on clinical and/or radiographic findings along with positive cultures from the affected organ (e.g., lungs, urinary tract, biliary tract, musculoskeletal system) with or without GE or bacteremia.

Use of gastric acid-reducing agents included administration of $\mathrm{H} 2$-blockers or proton pump inhibitors within 30 days of infection. Immunosuppressive therapy included use of corticosteroids (high dose was defined as $\geq 600 \mathrm{mg}$ of prednisolone or its equivalent, per month), cytotoxic chemotherapy, a calcineurin inhibitor, or TNFalpha blockade within 30 days of infection.

Fever was defined as body temperature $>38{ }^{\circ} \mathrm{C}$. Neutropenia was defined as absolute neutrophil count $\leq 500$ cells $/ \mu \mathrm{L}$ and lymphopenia as an absolute lymphocyte count $\leq 500$ cells $/ \mu \mathrm{L}$. Splenomegaly was defined based on radiographic findings as spleen measurement $>11 \mathrm{~cm}$.

Sepsis was defined as systemic inflammatory response syndrome caused by infection with $\geq 2$ of the following findings: pulse rate $>90$ beats/minute, respiratory rate $>20$ breaths/minute, body temperature $>38{ }^{\circ} \mathrm{C}$ or $<36{ }^{\circ} \mathrm{C}$, or white blood cell count of $>12,000 / \mu \mathrm{L}$ or $<4000 / \mu \mathrm{L}$. Severe sepsis was defined as sepsis with 
hypotension responsive to fluid boluses. Septic shock was defined as hypotension not responsive to fluid boluses, requiring use of vasopressors and/or accompanied by organ failure [13].

\section{Results}

We identified from the microbiological records 112 isolates of NTS collected between January 1, 2000, and December 31, 2013, corresponding to 84 individual patients. Two patients with one isolate each did not have a diagnosis of cancer and were excluded. The remaining 110 isolates corresponded to 82 cancer patients with 88 episodes of NTS infection, including five relapses $(6 \%)$ in four patients and a second consecutive episode of infection in one patient (the first infection with serotype $\mathrm{C} 1$ and the second with serotype F).

\section{Patient demographics}

Demographic details are shown in Table 1.

\section{Microbiological findings}

Among the 110 isolates, 51 (46\%) were obtained from blood cultures, 27 (25\%) from stool, 19 (17\%) from urine, and $13(12 \%)$ from other sites. The most common serogroup isolated was C, with 38 isolates (35\%), followed by B with 28 isolates (25\%), and D with 26 isolates (24\%). Serogroups E, F, G, and H comprised the remaining 18

Table 1 Demographic and clinical characteristics of patients with non-typhoidal Salmonella infection ( $\mathrm{N}=82)$

\begin{tabular}{|c|c|}
\hline Characteristic & $\mathrm{N}(\%)$ \\
\hline Median age (range) & $\begin{array}{l}55 \text { years } \\
(1-83 \\
\text { years })\end{array}$ \\
\hline Female sex & $38(46)$ \\
\hline Hematologic malignancy & $55(67)$ \\
\hline Acute leukemia/Myelodysplastic syndrome & 18 \\
\hline Chronic leukemia & 8 \\
\hline Multiple myeloma & 9 \\
\hline Lymphoma & 20 \\
\hline Solid tumors & $27(33)$ \\
\hline Gastrointestinal & 7 \\
\hline Genitourinary & 5 \\
\hline Breast & 4 \\
\hline Lung & 4 \\
\hline Head and neck & 3 \\
\hline Nervous system & 3 \\
\hline Melanoma & 1 \\
\hline Hematopoietic stem cell transplantation & $14(17)$ \\
\hline Autologous & 7 \\
\hline Allogeneic & 7 \\
\hline
\end{tabular}

isolates (16\%). Almost all isolates were susceptible to the commonly prescribed antimicrobials against this infection. Eleven of 98 isolates tested (11\%) were resistant to ampicillin, 3 of 101 (3\%) to ciprofloxacin, and 4 of $100(4 \%)$ to trimethoprim/sulfamethoxazole. All isolates tested were susceptible to ceftriaxone, cefepime, and imipenem.

\section{Clinical characteristics}

The clinical characteristics of 88 episodes of NTS infection are presented in Table 2. Patient factors that impair host defenses included lymphopenia and neutropenia, present within 30 days prior to infection in $28 \%$ and $13 \%$ of episodes, respectively, and during infection in $52 \%$ and $25 \%$, respectively. Fifty-six percent of patients took medications that reduced gastric acid secretion, a barrier to Salmonella infection. Medications that impaired the immune response, such as corticosteroids $(49 \%)$ or active chemotherapy and other immunosuppressants (66\%), were also common within 30 days of an infection episode. Patients had received antibiotics within 30 days prior to a positive culture in $40 \%$ of

Table 2 Clinical characteristics of 88 episodes of non-typhoidal Salmonella infection

\begin{tabular}{lc}
\hline Clinical characteristics & N (\%) \\
\hline Host Factors & $11(13)$ \\
Neutropenia in previous 30 days & $22(25)$ \\
Neutropenia during infection & $25(28)$ \\
Lymphopenia in previous 30 days & $46(52)$ \\
Lymphopenia during infection & \\
Medications within 30 days of infection & $49(56)$ \\
Gastric acid suppression & $43(49)$ \\
Corticosteroids & $58(66)$ \\
Active chemotherapy & $35(40)$ \\
Antibiotics & \\
Clinical presentation & $51(58)$ \\
Fever & $48(55)$ \\
Diarrhea & $40(45)$ \\
Nausea/vomiting & $37(42)$ \\
Abdominal pain & $9(10)$ \\
Urinary symptoms & \\
Sepsis syndrome & $32(36)$ \\
Sepsis & $24(27)$ \\
Severe sepsis & $5(6)$ \\
Septic shock & \\
Outcomes & $64(73)$ \\
Hospital admission & $11(13)$ \\
Intensive Care Unit admission & $3(3)$ \\
Mechanical ventilation & $7(8)$ \\
All-cause mortality at day 30 &
\end{tabular}


episodes, including long term antibiotic prophylaxis at the time of diagnosis in 11 episodes (13\%), of which four each received fluoroquinolones and trimethoprim/ sulfamethoxazole and three cefpodoxime. We could not verify compliance with prophylaxis from the chart. Only one of the 11 isolates was resistant to the prophylactic antibiotic being prescribed (trimethoprim/ sulfamethoxazole).

The most common symptoms at presentation included fever (58\%), diarrhea (55\%), nausea/vomiting (45\%), abdominal pain (42\%), and urinary symptoms, such as dysuria, urinary frequency, or lower back pain (10\%). Most patients had sepsis syndrome (69\%), with $33 \%$ presenting with severe sepsis or septic shock, occurring with all clinical presentations of NTS (Table 3).

Table 3 shows the episodes of infection classified according to previously defined clinical syndromes. Twelve of the 34 episodes (35\%) with acute GE had bacteremia, whereas 22 of 88 episodes (25\%) had disease limited to the gastrointestinal tract. There were $27 \mathrm{epi-}$ sodes of primary bacteremia and 27 of focal infection. The most serious episodes of focal infection included five cases of musculoskeletal infection, four of them with bacteremia but none with acute GE; two cases of pneumonia, one of them with acute GE; and one case of bone marrow infection that relapsed and required prolonged intravenous therapy.

Urinary tract infection (UTI) was the most common focal infection: sixteen patients had 18 episodes, including one relapse and one with consecutive infections with different strains of NTS. Six of these patients (38\%) had one or more urinary tract abnormalities or a foreign body, including nephrolithiasis (4), hydronephrosis (3), and one each with history of recurrent UTIs, urinary incontinence, vesicoureteral reflux, ureteral stent, and placement of an Indiana pouch. Only two of them had acute GE and none had bacteremia.

Table 3 Occurrence of severe sepsis and deaths by clinical presentation

\begin{tabular}{llll}
\hline Clinical presentation & \multicolumn{2}{l}{ No. (\%) } & \\
\cline { 2 - 4 } & Episodes & $\begin{array}{l}\text { Severe sepsis } \\
\text { or septic shock }\end{array}$ & $\begin{array}{l}\text { Death } \\
\text { within 30 } \\
\text { days }\end{array}$ \\
\hline Acute GE & $22(25)$ & $8(36)$ & $0(0)$ \\
Acute GE with bacteremia & $12(13)$ & $6(50)$ & $1(8)$ \\
Primary bacteremia & $27(31)$ & $10(37)$ & $2(7)$ \\
Focal infection & $27(31)$ & $5(19)$ & $4(15)$ \\
Total & $88(100)$ & $29(33)$ & $7(8)$ \\
\hline
\end{tabular}

GE, Gastroenteritis

\section{Management and outcomes}

Most episodes required hospitalization (73\%) and 13 required admittance to the intensive care unit, although only $3 \%$ required mechanical ventilation. Antimicrobial therapy was documented in 79 episodes (90\%), with a median duration of 14 days (range 1-61 days). Antibiotics were initiated the same day cultures were obtained in 64 of $79(81 \%)$ episodes. Initial therapy included cephalosporins in 34 episodes, fluoroquinolones in 24, carbapenems in 11, beta lactam/beta lactamase inhibitors in 9, and trimethoprim/sulfamethoxazole in 1. Appropriate antimicrobial therapy was started within $24 \mathrm{~h}$ of cultures being obtained in 35 of 43 (81\%) episodes of bacteremia and within $72 \mathrm{~h}$ in 40 of 43 (93\%) episodes.

Among nine episodes (10\%) with no antimicrobial therapy documented, one patient died of progressive brain cancer with intracranial hemorrhage the day the urine culture was obtained. One patient had bacteremia but was not hospitalized and the infection was regarded as a contaminant by the primary oncologist. Three patients had acute GE and five had UTI. It is not clear if these patients received therapy outside our institution. Surgical intervention with percutaneous drainage was performed in all four episodes with musculoskeletal abscess formation.

One patient with acute GE had two relapses, and one patient each with UTI, acute GE, and bone marrow infection had one relapse. Patients who had a relapse received a shorter course of antibiotic therapy for the initial infection with a median duration of 7 days (range 2-10 days), compared with a median of 14 days for all treated patients. Five of 20 episodes treated for $\leq 10$ days (25\%) resulted in a relapse compared with none in those treated for longer than 10 days. One patient had two episodes of UTI with different NTS serotypes (reinfection).

Seven patients died within 30 days of infection, all had invasive NTS disease, but four died of cancer progression and NTS was considered a contributing factor to death in the remaining three cases. Among these three patients, one died of disseminated fungal infection proven by autopsy, one of graft-versus-host disease causing hepatorenal failure, and one of polymicrobial bacteremia and sepsis that included NTS. NTS was considered one of the principal contributors to death only in the last case.

\section{Discussion}

Our data indicated that NTS continues to cause severe infections in cancer patients, particularly in those with hematologic malignancies [14]. Patients with cancer have an increased risk of acquiring NTS infection because of mucosal barrier disruption due to cancer or its therapy [15], immunodeficiency due to cancer itself and receipt 
of cytotoxic chemotherapy and/or corticosteroids [3, $14,16]$. Other previously described factors such as gastric acid suppression, use of antibiotics in the prior month, and old age were also highly prevalent among our patients $[2,4,8]$. Hematologic malignancies account for $10 \%$ of the annual incidence of cancer in the United States [17], however, they constituted two-thirds of our patients with NTS infection and $48 \%$ and $60 \%$ in previous case series [10, 11]. Gradel et al. reported that blood cancers might increase the risk of Salmonella infection by a factor of 5-10 when comparing patients in the Danish Cancer Registry with matched controls, whereas patients with solid tumors had an increased risk of only 1-1.5 times that of controls [18]. Blood cancers are associated with prolonged and profound cellular and humoral immunodeficiency. Improved survival and stem cell transplantation leading to long-term immunosuppression may increase chances of exposure and susceptibility to infection.

Among patients with solid tumors, upper or lower gastrointestinal malignancies were most common (26\%). These patients may have mucosal barrier disruption, lack of gastric acidity, and other anatomic or physiologic abnormalities that facilitate infection through this most common portal of entry. Genitourinary malignancies were second most common (19\%). This is not surprising, because structural abnormalities of the urinary tract caused by the cancer or its treatment, as well as nephrolithiasis, obstruction, and renal transplantation, have been previously described in most patients with NTS UTI [19], and these factors likely favor NTS infection of the urinary tract.

As expected, there was a wide distribution of NTS serogroups, given that patients most likely acquire the infection sporadically in the community. Our isolates showed low to moderate levels of antimicrobial resistance, in contrast to reports of increasing resistance in the United States [20]. Drug resistance has been associated with excess bloodstream infections and hospitalizations [21], including resistance rates to ceftriaxone of $2.9 \%$ between 1996 and 2013 [22].

One surprising finding was the occurrence of NTS infection in patients receiving long-term prophylactic antibiotics to which the organism was sensitive in 10 cases, however, we could not verify the total duration or compliance with these antibiotics. Possible reasons include noncompliance with antibiotic prophylaxis, breakthrough infection due to high inoculum, or severe immunosuppression, as well as partial effectiveness of the prophylaxis.

Most of our patients presented with sepsis, and onethird had severe sepsis or septic shock, with $13 \%$ requiring admission to the intensive care unit. GE, bacteremia, or both were present in $69 \%$ of patients, and the rest had focal infection, most commonly UTI. We found that four episodes included musculoskeletal infection that required abscess drainage.

The mortality rate in our series was lower than expected based on prior reports and severity of illness, with an overall 30-day mortality rate of $8 \%$. NTS infection contributed in three of the 7 patients who died (4\%) and was considered a significant contributor in one patient. Noriega et al. reported a higher 30-day mortality rate $(23 \%)$ and attributable mortality rate $(15 \%)$ among 40 patients with Salmonella infections in a cancer center in Belgium from 1975 to 1990 [10].

NTS bacteremia has high mortality rates even in modern times. Three of 43 bacteremic patients (27 with primary bacteremia, 12 with acute GE with bacteremia and 4 with musculoskeletal infection and bacteremia) in our series (7\%) died within 30 days. A study from Taiwan reported an in-hospital mortality rate for NTS bacteremia of $41 \%$ in patients with cancer and $18 \%$ in those without [8], and a Malaysian study reported an overall mortality rate of $31 \%$ in patients with cancer compared with $5 \%$ in others [5]. Owing to different definitions, these numbers are not directly comparable but serve as a reference. Patients with advanced cancer or its complications may have high mortality rates independent of NTS infection, as seen in our study.

Delay in initiating appropriate antibiotic therapy in patients with sepsis and septic shock is associated with increased mortality [23]. Yen el al reported that inadequate antibiotic treatment of NTS bacteremia was an independent risk factor predicting mortality by multivariate logistic regression analysis [9]. Only $71 \%$ and $62 \%$ of patients with NTS bacteremia in two studies received appropriate antimicrobials within $72 \mathrm{~h}[5,8]$, compared to $81 \%$ (35 of 43 ) of our bacteremic patients receiving appropriate antimicrobials within $24 \mathrm{~h}$ and $93 \%$ (40 of 43) within 72 h. Empiric antimicrobial therapy for cancer patients with sepsis admitted to our institution includes third- or fourth-generation cephalosporins or carbapenems (no NTS resistance found in our study). The use of early and appropriate empiric antibiotic therapy may have contributed to improved outcomes.

The recommended duration of therapy for NTS GE in immunosuppressed individual is 3-7 days, 10-14 days for NTS bacteremia, and longer duration (4-6 weeks) is recommended for complicated infections such as musculoskeletal (plus surgical drainage, if indicated), endarteritis, or endocarditis [16]. One quarter (5 of 20) of our patients who received antibiotic therapy for $\leq 10$ days relapsed, compared with none of the 68 treated for more than 10 days. Our cohort was not large enough to allow statistical comparisons; however, these findings suggest that a 
longer course of therapy for NTS may be needed to prevent relapse in patients with cancer. Noriega et al. reported that five of six patients with Salmonella infections in a cancer center in Belgium who experienced relapse received less than 10 days of therapy [10].

Our study had several limitations. It was based in a comprehensive cancer center were the severity of illness and predisposing factors such as degree of immunosuppression may be higher than in patients with cancer in the community. Secondly, its retrospective nature and the number of patients relative to the number of variables to consider did not allow for certain statistical comparisons that may have given more strength to our conclusions.

\section{Conclusions}

NTS is a cause of severe infections in cancer patients, particularly in those with hematologic malignancies, followed by patients with gastrointestinal and genitourinary cancers. Invasive disease, sepsis, and septic shock are common among admitted patients. Antimicrobial prophylaxis may not prevent NTS infection. Thirty-day mortality and attributable mortality rates were low in our series compared to older case series. Early appropriate antibiotic therapy may have had a role in decreasing mortality. Relapses occurred in patients receiving $\leq 10$ days of therapy, suggesting the need for longer duration of antibiotic therapy in patients with cancer and uncomplicated NTS infections.

\section{Abbreviations}

NTS: Non-typhoidal Salmonella; GE: Acute gastroenteritis; TNF: Tumor necrosis factor; UTI: Urinary tract infection.

\section{Acknowledgements}

Erica Goodoff of Scientific Publications, Research Medical Library, University of Texas MD Anderson Cancer Center, provided editorial assistance.

\section{Authors' contributions}

Conception and design: NM, ADS, and VEM. Acquisition and assembly of data: NM, JJT, and VEM. Data analysis and interpretation: NM, ADS, JAA, JJT and VEM. Manuscript writing: NM, ADS, and VEM. Final approval of manuscript: NM, ADS, JAA, JJT and VEM. All authors read and approved the final manuscript.

\section{Funding}

This work was supported in part by funds from The University of Texas MD Anderson Cancer Center, Houston, Texas; and the National Cancer Institute at the National Institutes of Health [P30CA016672]. The funding institutions had no role in the design of the study and collection, analysis, and interpretation of data and in writing the manuscript.

\section{Availability of data and materials}

The datasets generated and/or analyzed during the current study are not publicly available due to patient confidentiality but are available from the corresponding author on reasonable request.

\section{Declarations}

\section{Ethics approval and consent to participate}

The study was conducted at The University of Texas MD Anderson Cancer Center in Houston, Texas, after approval by its Institutional Review Board.
Informed consent waiver was granted because of the retrospective, noninterventional nature of the study.

\section{Consent for publication}

Not applicable.

\section{Competing interests}

On behalf of all authors, the corresponding author states that there is no conflict of interest.

\section{Author details}

${ }^{1}$ Department of Infectious Diseases, Infection Control and Employee Health, The University of Texas MD Anderson Cancer Center, 1515 Holcombe Boulevard, Unit 1460, Houston, Texas 77030, USA. ²Department of Laboratory Medicine, The University of Texas MD Anderson Cancer Center, 1515 Holcombe Boulevard, Unit 0084, Houston, Texas 77030, USA. ${ }^{3}$ Present Address: Division of Infectious Diseases, St. Luke's International Hospital, 9-1 Akashi-cho, Chuo-ku, Tokyo 104-8560, Japan.

Received: 13 December 2020 Accepted: 17 September 2021

Published online: 29 September 2021

\section{References}

1. Powell MR, Crim SM, Hoekstra RM, Williams MS, Gu W. Temporal patterns in principal Salmonella serotypes in the USA; 1996-2014. Epidemiol Infect. 2018;146(4):437-41.

2. Delarocque-Astagneau E, Bouillant C, Vaillant V, Bouvet P, Grimont PA, Desenclos JC. Risk factors for the occurrence of sporadic Salmonella enterica serotype typhimurium infections in children in France: a national case-control study. Clin Infect Dis. 2000;31(2):488-92.

3. Gordon MA. Salmonella infections in immunocompromised adults. J Infect. 2008;56(6):413-22.

4. Leonard J, Marshall JK, Moayyedi P. Systematic review of the risk of enteric infection in patients taking acid suppression. Am J Gastroenterol. 2007;102(9):2047-56 (quiz 2057).

5. Dhanoa A, Fatt QK. Non-typhoidal Salmonella bacteraemia: epidemiology, clinical characteristics and its' association with severe immunosuppression. Ann Clin Microbiol Antimicrob. 2009;8:15.

6. Han T, Sokal JE, Neter E. Salmonellosis in disseminated malignant diseases. a seven-year review (1959-1965). N Engl J Med. 1967;276(19):1045-52.

7. Cherubin CE, Fodor T, Denmark LI, Master CS, Fuerst HT, Winter JW. Symptoms, septicemia and death in salmonellosis. Am J Epidemiol. 1969;90(4):285-91.

8. Li CW, Chen PL, Lee NY, Lee HC, Chang CM, Lee CC, Ko WC. Non-typhoidal Salmonella bacteremia among adults: an adverse prognosis in patients with malignancy. J Microbiol Immunol Infect. 2012;45(5):343-9.

9. Yen YF, Wang FD, Chiou CS, Chen YY, Lin ML, Chen TL, Liu CY. Prognostic factors and clinical features of non-typhoid Salmonella bacteremia in adults. J Chin Med Assoc. 2009;72(8):408-13.

10. Noriega LM, Van der Auwera P, Daneau D, Meunier F, Aoun M. Salmonella infections in a cancer center. Support Care Cancer. 1994;2(2):116-22.

11. Wolfe MS, Louria DB, Armstrong D, Blevins A. Salmonellosis in patients with neoplastic disease. A review of 100 episodes at Memorial Cancer Center over a 13-year period. Arch Intern Med. 1971;128(4):546-54.

12. Ramos JM, Garcia-Corbeira P, Aguado JM, Ales JM, Soriano F. Classifying extraintestinal non-typhoid Salmonella infections. QJM. 1996;89(2):123-6.

13. Bone RC, Balk RA, Cerra FB, Dellinger RP, Fein AM, Knaus WA, Schein RM, Sibbald WJ. Definitions for sepsis and organ failure and guidelines for the use of innovative therapies in sepsis. The ACCP/SCCM Consensus Conference Committee. American College of Chest Physicians/Society of Critical Care Medicine. Chest. 1992;101(6):1644-55.

14. Shimoni Z, Pitlik S, Leibovici L, Samra Z, Konigsberger H, Drucker M, Agmon V, Ashkenazi S, Weinberger M. Nontyphoid Salmonella bacteremia: age-related differences in clinical presentation, bacteriology, and outcome. Clin Infect Dis. 1999;28(4):822-7.

15. Delaloye J, Merlani G, Petignat C, Wenger A, Zaman K, Monnerat C, Matzinger O, Beck Popovic M, Vuichard P, Ketterer N, et al. Nosocomial nontyphoidal salmonellosis after antineoplastic chemotherapy: 
reactivation of asymptomatic colonization? Eur J Clin Microbiol Infect Dis. 2004;23(10):751-8.

16. Hohmann EL. Nontyphoidal salmonellosis. Clin Infect Dis. 2001;32(2):263-9.

17. Siegel RL, Miller KD, Jemal A. Cancer statistics, 2020. CA Cancer J Clin. 2020;70(1):7-30.

18. Gradel KO, Norgaard M, Dethlefsen C, Schonheyder HC, Kristensen B, Ejlertsen T, Nielsen H. Increased risk of zoonotic Salmonella and Campylobacter gastroenteritis in patients with haematological malignancies: a population-based study. Ann Hematol. 2009;88(8):761-7.

19. Cohen JI, Bartlett JA, Corey GR. Extra-intestinal manifestations of salmonella infections. Medicine. 1987;66(5):349-88.

20. Crump JA, Medalla FM, Joyce KW, Krueger AL, Hoekstra RM, Whichard JM, Barzilay EJ, Emerging Infections Program NWG. Antimicrobial resistance among invasive nontyphoidal Salmonella enterica isolates in the United States: National Antimicrobial Resistance Monitoring System, 1996 to 2007. Antimicrob Agents Chemother. 2011;55(3):1148-54.
21. Varma JK, Molbak K, Barrett TJ, Beebe JL, Jones TF, Rabatsky-Ehr T, Smith KE, Vugia DJ, Chang HG, Angulo FJ. Antimicrobial-resistant nontyphoidal Salmonella is associated with excess bloodstream infections and hospitalizations. J Infect Dis. 2005;191(4):554-61.

22. Iwamoto M, Reynolds J, Karp BE, Tate H, Fedorka-Cray PJ, Plumblee JR, Hoekstra RM, Whichard JM, Mahon BE. Ceftriaxone-resistant nontyphoidal Salmonella from humans, retail meats, and food animals in the United States, 1996-2013. Foodborne Pathog Dis. 2017;14(2):74-83.

23. Rhodes A, Evans LE, Alhazzani W, Levy MM, Antonelli M, Ferrer R, Kumar A, Sevransky JE, Sprung CL, Nunnally ME, et al. Surviving sepsis campaign: international guidelines for management of sepsis and septic shock: 2016. Crit Care Med. 2017:45(3):486-552.

\section{Publisher's Note}

Springer Nature remains neutral with regard to jurisdictional claims in published maps and institutional affiliations.
Ready to submit your research? Choose BMC and benefit from:

- fast, convenient online submission

- thorough peer review by experienced researchers in your field

- rapid publication on acceptance

- support for research data, including large and complex data types

- gold Open Access which fosters wider collaboration and increased citations

- maximum visibility for your research: over $100 \mathrm{M}$ website views per year

At BMC, research is always in progress.

Learn more biomedcentral.com/submissions 